\title{
Beiträge zur Kenntnis der Tellursäure.
}

$$
\text { Von }
$$

\author{
A. Gutbier.
}

In einer vorläufigen Mitteilung ${ }^{1}$ habe ich vor kurzem die Befunde mitgeteilt, welche ich bei meinen Untersuchungen über die Tellursäure erhielt, und beabsichtige im folgenden ausführlich die Resultate zu publizieren.

Bekanntlich besteht die einzige Möglichkeit, zu chemisch reinem Tellur zu gelangen, in der Reduktion der Tellursäure, nachdem es Straddenmaier $^{2}$ gelungen ist, durch die Eutdeckung einer neuen Darstellungsmethode die Süure bequem und in jedem beliebigen Quantum zu erhalten. Die Tellursäure ist schon lange bekannt und zuerst von Berzelius, ${ }^{3}$ nach diesem von Oppenterm ${ }^{4}$ und schliefslich von F. BECKER dargestellt und beschrieben worden. Die Darstellungsmethoden genannter Forscher hatten aber den Nachteil, dafs dic entstehenden Tellurate (Baryum- oder Bleisalze) mit Schwefelsäure zersetzt werden mufsten, welch letztere sich aber nur schwierig und fast nie vollkommen entfernen liefs, so dafs sie der Tellursäure selbst nach häufigem Umkrystallisieren beigemengt blieb, und die Krystalle infolgedessen nicht den ihnen zukommenden Glanz zeigten. Um diesen Übelstand zu beseitigen, versuchte Staudenmaier nach der Thomsen'schen Selensäuredarstellung mit gutem Erfolge die Gewinnung der Tellursäure durch die Einwirkung von Brom auf tellurigsaures Silber bei Gegenwart von Wasser zu bewerksteligen; er verliels aber diese umständliche Methode bald.

1 Ber. deutseh. chem. Ges. 34, 2114.

2 Z. anoig. Chem. 10, $189 \mathrm{ff}$.

${ }^{3}$ Lehrbuch, 5. Aufl.; II, 241.

4 Journ. prakt. Chem. 71, 266.

s Ann. Chem. 180, 267. 
nachdem es ihm gelungen war, eine direkte Uberführung des Tellurs und seiner Mineralien in Tellursäure - durch Oxydation mittels Chromsäure in salpetersaurer Lösung -, nach der Gleichung:

$$
3 \mathrm{TeO}_{2}+2 \mathrm{CrO}_{3}+3 \mathrm{H}_{2} \mathrm{O}=3 \mathrm{H}_{2} \mathrm{TeO}_{4}+\mathrm{Cr}_{2} \mathrm{O}_{3}
$$

zu erzielen.

Auf die Methode selbst, welche ich sehr empfehlen kann man erhält aus $1 \mathrm{~kg} 36 \%$ igem ungarischen Rohtellur ca. $3 /{ }_{4} \mathrm{~kg}$ reine Tellursäure - will ich hier nicht näher eingehen; sie ist so gut beschrieben, dals sie keiner weiteren Erläuterung bedarf; einige kritische Bemerkungen dürften aber wohl am Platze sein.

Die Ansicht StaddenMaIER's, dals man jetzt wohl in der Lage sein könnte, das ungarische Rohtellur direkt auf Tellursäure zu verarbeiten, nachdem in Selmeczbánya das Tellur jetzt nicht mehr mit Zink, sondern mit schwefliger Säure gefällt werde, kann ich nicht teilen. Ich habe bei meinen ersten Versuchen die Erfahrung gemacht, dafs die Nitrate und Oxyde der übrigen, im Rohtellur vorkommenden Metalle ziemlich störend auf den Darstellungsgang einwirken, und dafs daher die kleine Mühe, das Tellur vor der Oxydation des Materiales mit Schwefeldioxyd zu fällen, eher anf sich zu nehmen ist, als nachber die grofse Schwierigkeit, die Tellursäure bei dem Eindampfen auf dem Wasserbade von diesen, in der salpeter-chromsauren Lösung meist schmierige Massen bildenden Verunreinigungen $\mathrm{zu}$ befreien.

Aufserdem habe ich gefunden, dafs man, um das ziemlich unangenehme Arbeiten mit den grofsen Mengen von konzentrierter Salpetersäure zu vermeiden, das Chromnitrat und die überschüssige Chromsäure ganz gut durch fraktionierte Fällung mit stark rerdünntem Ammoniak entfernen kann. Operiert man recht vorsichtig, und lälst man aus einem Tropftrichter nur gerade soviel Ammoniak zuflielsen, bis eben noch ein gefärbter Niederschlag entsteht, so braucht man dann nur abzufiltrieren, den Niederschlag mit heifsem Wasser ordentlich auszuwaschen und dann das Filtrat mitsamt dem Waschwasser auf dem Wasserbade bis zur beginnenden Krystallisation einzudampfen. Nach diesem Verfahren verarbeitete ich einzelne Rückstände, aus denen ich eine Säure erhielt, die nach mehrmaligem Umkrystallisieren genau so rein war, als die auf die andere Weise erhaltene.

Die Methode, die Tellursäure in Gestalt ihres Baryumsalzes zu isolieren, ist meiner Ansicht nach - abgesehen von den oben ge- 
schilderten unangenehmen Eigenschaften der zur Zersetzung notwendigen Schwefelsäure - schon deshalb durchaus zu verwerfen, weil das Baryumtellurat sowohl in Wasser" als auch in Ammoniak viel zu leicht löslich ist, als dafs eine quantitative Abscheidung erfolgen könnte.

Die Tellursäure, unter welchem Namen man bekanntlich die Säure von der Zusammensetzung $\mathrm{H}_{6}{ }^{\prime} \mathrm{IeO}_{6}=\mathrm{H}_{2}{ }^{2} \mathrm{TeO}_{4}+2 \mathrm{H}_{2} \mathrm{O}$ versteht, krystallisiert äufserst leicht und gut, so dafs sie in grolsen, wasserklaren Krystallen erhalten werden kann. Fine Analyse, mit welcher ich mich von der Reinheit meines Produktes überzeugte, ergab folgendes:

$1.4777 \mathrm{~g}$ exsikkatortrockener Substanz hinterlielsen beim Fällen mit Natriumbisulfitlösung: $0.8234 \mathrm{~g}$ Tellur.

Ber. für $\mathrm{H}_{6} \mathrm{TeO}_{6}: 55.56 \%$ Te. Gef. $55.72 \%$ Te.

Ein zweites Hydrat, dem die Formel $\mathrm{H}_{2} \mathrm{TeO}_{4}+6 \mathrm{H}_{2} \mathrm{O}=$ $\mathrm{H}_{6} \mathrm{TeO}_{6}+4 \mathrm{H}_{2} \mathrm{O}$ zukommt, hat StaUdenMareR ${ }^{1}$ durch Abkühlung der wässerigen Lösung bis auf ca. $0^{0}$ zuerst erhalten; es eignet sich recht gut zur Reinigung der Tellursäure, da es die Eigenschaft besitzt, durch geringe Erhöhung der Temperatur, wuzu schon die Handwärme genügt, in ein aufserordentlich feines Aggregat der Säure $\mathrm{H}_{6} \mathrm{TeO}_{6}$ überzngehen.

Die von Benzelús ${ }^{2}$ beschriebene Säure $\mathrm{H}_{2} \mathrm{TeO}_{4}$, die er als Tellursäure b bezeichnet, läfst sich unter keinen Umständen erbalten, bezw. isolieren. Ich habe verschiedentlich die Darstellung dieses Produktes versucht, aber stets ohne Erfolg. Die einzige und zugleich auch einfachste Methode, um zur Säure $\mathrm{H}_{2}{ }^{\mathrm{TeO}} \mathrm{O}_{4}$ zu gelangen, würde darin bestehen, dals man durch vorsichtiges Erhitzen der Süure $\mathrm{H}_{6} \mathrm{TeO}_{6}$ die beiden, ihr eigentümlichen Moleküle Wasser entrieht, und hierbei als Endprodukt die Säure $\mathrm{H}_{2} \mathrm{TeO}_{4}$ erhält.

Angestellte Versuche ergaben jedoch, dals es praktisch unmöglich ist, eine Wasserbestimmung der Tellursäure und somit eine Entwässerung durch einfuches Erhitzen der Substanz bis zu konstantem Gewichte auszuführen, da die Tellursäure sich hierbei yersetzt, d. h. Sauerstoff mit abgiebt. Man mufs also zur Bestimmung des Wassergehaltes einen umständlicheren Weg einschlagen, inden man die Säure in einem Strome trockener Luft erhitzt und

\footnotetext{
1. c., S. 192 .

$\because$ l. c. Il, s. 238 if.
} 
das entweichende Wasser in einem Chlorealciumrohre auffängt. Die Resultate dieser Bestimmungen werde ich in einer späteren Publikation angeben, da sie vor allen Dingen den Zweck hatten, zu prüfen, ob die Tellursäure für Zwecke der Atomgewichtsbestimmungen ${ }^{1}$ anwendbar sei oder nicht, und hier nur Angaben über das Verhalten der Tellursäure beim Erhitzen im Platintiegel machen.

Nach Berzelius ${ }^{2}$ soll die Säure $\mathrm{H}_{6} \mathrm{~T}^{\top} \mathrm{O}_{6}$ bei etwa $100^{\circ}$ beide Moleküle Wasser verlieren und sich dabei unter Beibehaltung ihrer Krystallform in eine schwach gelbe, in Wasser nur bei anhaltendem Kochen lösliche Masse verwandeln, die der Formel $\mathrm{H}_{2} \mathrm{TeO}_{4}$ entsprechen soll.

Dals dem nicht so sei, bemerkte ich schon, als ich bei der Darstellung der Tellursäure das noch mit Salpetersäure verunreinigte Präparat im Trockenschranke erhitzte und hierbei die Temperatur versehentlich auf $100^{\circ}$ gestiegen und längere Zeit geblieben war; die Säure hatte nicht nur ihre Krystallform, sondern auch ibre Farbe vollständig beibehalten und löste sich ganz leicht in heifsem Wasser, aus dem sie damn in der gewöhnlichen Form wieder auskrystallisierte; allerdings verliert die Säure bei dieser Temperatur schon einen Teil ihres Wassers, aber bei weitem nicht eine Menge, die der für die beiden Moleküle berechneten nur irgendwie nahe kommen dürfte.

Ich verfuhr nun zur Ausführung der Versuche folgendermafsen:

$0.9880 \mathrm{~g}$ reinste, exsikkatortrockene Säure $\mathrm{H}_{6} \mathrm{TeO}_{6}$, welche vor dem Trocknen zur Entfernung etwa noch anhaftender Mutterlauge mit reinem destilliertem Wasser gewaschen worden war, wurden in einem gewogenen Platintiegel nach und nach auf höhere Temperaturen erhitzt, wobei mit grölster Vorsicht versucht wurde, bei den $100^{\circ}$ nur wenig übersteigenden Temperaturen Gewichtskonstanz zu erhalten. Es stellte sich aber hald heraus, dafs dies so gut wie unmöglich ist, da die Säure beim Erhitzen über $100^{\circ}$ langsam, aber stetig ibr Wasser verliert, bis der Rückstand bei $145^{\circ}$ Gewichtskonstanz zeigt.

Bei ca. $1 / 2$ stündigem Erhitzen auf $110-115^{0}$ hatte die angewandte Substanzmenge $0.0790 \mathrm{~g}$ an Gewicht abgenommen, was ungefähr dem für $1 \mathrm{Mol}$. berechneten Werte entspricht, da $8.00 \%$ $\mathrm{H}_{2} \mathrm{O}$ gefunden und $7.84 \% \mathrm{H}_{2} \mathrm{O}$ berechnet wurden.

1 Es sei hier andeutungsweise bemerkt, dal's ich für das Tellur ein Atomgewicht von $127.51(0=16, \mathrm{H}=1.008)$ gefunden habe.

1. c., s. $238 \mathrm{ft}$. 
Bei weiterem Erhitzen auf die gleiche Temperatur nahm das Gewicht noch um weitere $0.0113 \mathrm{~g}=1.14 \% \mathrm{ab}$, so dals der Wasserverlust der Tellursäure bei $110-115^{\circ} 9.14^{\%}$ beträgt. Steigert man nun die Temperatur weiter, so findet eine erneute Wasserabgabe statt, bis bei $145^{\circ}$ Gewichtskonstanz erfolgt. Der hierbei gefundene Gewichtsverlust entspricht aber keineswegs dem für $2 \mathrm{H}_{2} \mathrm{O}$ berechneten, denn $0.9880 \mathrm{~g} \mathrm{H}_{6} \mathrm{TeO}_{6 \mathrm{~s}}$ verloren nur $0.1213 \mathrm{~g}=12.30 \%$, während die Theorie $15.68 \% \mathrm{H}_{2} \mathrm{O}$ verlangen würde.

Wird die Temperatur nun noch mehr erhöht, so findet die weitere Vertreibung des Wassers nur unter Zersetzung der Tellursäure statt, indem bei derjenigen Temperatur, die gerade genügen dürfte, um die letzten Anteile des Wassers zu entfernen, auch schon Sauerstoff mitentweicht, wobei sich die Säure in eine gelbliche Masse verwandelt, die ein Gemisch von Tellursäure mit Tellurtrioxyd und T'ellurdioxyd bildet.

Die ausgeführte Bestimmung ergab nämlich, dals $0.9880 \mathrm{~g}$ $\mathrm{H}_{6} \mathrm{TeO}_{6}$ beim Erhitzen bis auf $170^{\circ}$ einen Gewichtsverlust von $0.1679 \mathrm{~g}=17.01 \%$ erlitten hatte, während letzterer, nach der Berechnung, beim Übergang von $\mathrm{H}_{6} \mathrm{TeO}_{6}$ in $\mathrm{H}_{2} \mathrm{TeO}_{4}$ nur $15.68 \%$ betragen sollte, und sich für den Übergang von $\mathrm{H}_{6} \mathrm{TeO}_{6}$ in $\mathrm{TeO}_{3}$ $23.52 \%$ Gewichtsverlust berechnen.

Brauner ${ }^{1}$ teilt allerdings mit, dals er die gelbe Tellursäure erhalten habe, indessen glaube ich nach meinen Befunden, dals auch er nur ein Gemisch der Tellursäure mit Tellurtrioxyd unter den Händen gehabt haben dürfte; nebenbei sei noch erwähnt, dafs auch das Anhydrid der Tellursäure praktisch nicht rein erhalten werden kann, da auch das Tellurtrioxyd mit beispielloser Leichtigkeit Sauerstoff abgiebt und stets mit mehr oder weniger Tellurdioxyd verunreinigt ist.

Als Endprodukt beim Erhitzen der Tellursäure hinterbleibt rein weilses Tellurdioxyd, zu dessen Gewinnung die Hitze einer Bunsenflamme nötig ist; hierbei verloren $0.9880 \mathrm{~g} \mathrm{H}_{6} \mathrm{TeO}_{6}$ beim Erhitzen bis zur Gewichtskonstanz $0.3013 \mathrm{~g}=30.48 \%$, während sich für den Übergang von $\mathrm{H}_{6} \mathrm{TeO}_{6}$ in $\mathrm{TeO}_{2}: 30.51 \%$ berechnen.

Die Tellursäure ist dimorph und 1 . in regulären Oktaëdern und 2. in einer Modifikation krystallinisch zu erhalten, welche von

1 Chem. Soc. Journ. 67 (1895), 550. 
den älteren Forschern zu dem monoklinen Systeme gerechnet wurden, während nach Staudenmaine's' ${ }^{1}$ Angaben W. Muthmann ihre Zugehörigkeit zum trigonalen Systeme bestimmt hatte.

Ich habe diese Angaben in Gemeinschaft mit Hrn. Dr. L. FinckH nachgeprïft und gefunden, dafs hier ein Irrtum vorliegt. Es gelang uns nämlich, nachzuweisen, dals die neben der regulären bestehende zweite Modifikation weder dem monoklinen, noch dem trigonalen, sondern dem hexagonal-rhomboëdrischen Krystallsysteme angehören.

Wie Herr O. Bnunck-Freiberg mir auf meine vorläufige Mitteilung hin brieflich mitteilte, hat er in Gemeinschaft mit WerssBACH ebenfalls vor längerer Zeit eine krystallographische Untersuchung der Tellursäure ausgeführt und ist $\mathrm{zu}$ den gleichen Resultaten wie ich gelangt. Da Herr BnUNCK eine ausführliche Veröffentlichung seiner Resultate in Aussicht gestellt hat, kann ich darauf verzichten, und ich will nur bemerken, dafs man der hexagonal-rhomboëdrischen Krystallform weit öfter als der regulären begegnet und sie namentlich aus verdünnten wässerigen Lösungen erhält; die Krystalle behalten ihren Glanz - auch bei längerem Aufbewahren an der Luft - dauernd, zeigen parallel der Prismenkanten auf allen $6 \infty 0$ R-Flächen gerade Auslöschung, und als häufigste Form $\infty R$ und $R$ als Abstumpfung der Prismen, während seltener OR als Basis auftritt.

Die Tellursäure ist eine schwache Säure und besitzt einen metallischen, nicht sauren Geschmack; in kaltem, sowie heifsem Wasser ist sie in allen Verhältnissen löslich, unlöslich dagegen in absolutem Alkohol, durch welch letzteren sie aus der wässerigen Lösung als feines mikrokrystallinisches Pulver ausgefällt wird; aus stark konzentrierten Lösungen scheidet sich die Säure in kompakten, zusammenhängenden Krystallkuchen ab, während sie aus verdünnten Lösungen bei langsamer Verdunstung des Wassers in wohlausgebildeten, vollkommen farblosen und durchsichtigen Einzelkrystallen erhalten wird, die die angenehme Eigenschaft haben, durch Mutterlaugeneinschlüsse nicht so stark verunreinigt zu sein, als die kompakten, strahlenförmig zusammengewachsenen Krystallisationen der konzentrierten Lösungen. Auch beim raschen Eindampfen der Säure erhielt ich, entgegen den Angaben älterer Forscher, keine glasigen

\footnotetext{
${ }^{1}$ l. c. S. 191.
} 
Massen, sondern ein krystallinisches Produkt, allerdings unter der Voraussetzung, dafs die Lösung einige Male mit einem Glasstabe umgerührt wurde.

Wie eben erwähnt, ist die Tellursäure eine schwache Säure; sie rötet blanes Lackmuspapier erst nach längerer Einwirkung sehr wenig und lälst sich, wie ich zeigen werde, unter Anwendung eines Indikators nicht titrieren.

Da nun nach den neueren Anschauungen ${ }^{1}$ die Stärke einer Säure, d. h. ihr Säurecharakter, durchaus nicht von der Beständigkeit der Verbindung selbst, sondern nur von dem Grade der Ionenbildung abhängt, unternahm ich es, zur experimentellen Prüfung des Säurecharakters der Tellursäure, Leitfähigkeitsbestimmungen der Säure $\mathrm{H}_{6} \mathrm{TeO}_{6}$ und ihres neutralen Kaliumsalzes $\mathrm{K}_{2} \mathrm{TeO}_{4}+5 \mathrm{H}_{2} \mathrm{O}$, welch letzteres am leichtesten rein zu erhalten ist und von allen Telluraten die bei weitem gröfste Löslichkeit in Wasser besitzt, anzustellen, indem ich für die Tellursäure selbst die molekulare, für das Kaliumtellurat die äquivalente Leitfähigkeit mafs.

Bei diesen Messungen ${ }^{2}$ fand ich, dafs die Tellursäure in gelöstem Zustande - als Lösungsmittel wurde reinstes destilliertes Wasser angewandt - nur in ganz geringem Grade dissoziiert ist, da der bestimmbare Maximalwert bei einer 32 fachen Verdünnung noch nicht einmal den Wert 0.5 für die molekulare Leitfähigkeit der Tellursäure ergab. Hieraus lälst sich schliefsen, dafs die Tellursäure in Lösung, genau wie Schwefelwasserstoff, Kohlensäure und Borsäure, für welche bekanntlich auch sehr kleine Werte gefunden worden sind, nur zum kleinsten Teile in Ionen gespalten ist, während die Hauptmenge als nicht dissoziierte Säure in Lösung geblieben ist.

Das zu den Messungen verwendete Wasser wurde durch anhaltende Destillation von destilliertem und über Ätzkalk stehengelassenem Wasser, welches aus einem Destillationsgefäls durch eine Kühlschlange aus Zinn abdestilliert wurde, gewonnen und hierbei nur das zweite Drittel des Destillates aufgefangen, welches eine Leitfähigkeit ron $0.5 \cdot 10^{-6}$ bis höchstens $1 \cdot 10^{-6}$ besals.

1 Ostwald, Grundlinien d. anorg. Chem., S. 241.

2 Ieh hatte mich bei den physikalisch-chemischen Bestimnungen der ausgezeichneten Hilfe des Herrn Privatdozent Dr. Hower zu erfrenen, dem ich auch an dieser Stelle für seine mir bewiesene liebenswürdigkeit meinen verbindlichsten Dank ausspreche. 
Die Bestimmungen selbst wurden in einem rauchfreien Zimmer bei einer Temperatur von $25^{\circ}$ ausgeführt, welch letztere durch ein von Herrn HoFER konstruiertes elektrisches Rührwerk mit elektrischer Heizung konstant erhalten wurde.

\section{Bestimmung der molekularen Leitfähigkeit der Tellursäure.}

1. Benutzt wurde ein Präparat der 'T'ellursäure, welches nach der üblichen Reinigung noch $5 \mathrm{mal}$ in einer Platinschale umkrystallisiert und vor dem Trocknen im Exsikkator noch durch Waschen mit reinstem destillierten Wasser von etwa anhängender Mutterlauge befreit worden war.

2. $\mathrm{Zu}$ den einzelnen Bestimmungen wurden je $20 \mathrm{ccm}$ von $1 / \mathrm{s}^{-}$ norm. Tellursäurelösung angewandt, d. h. einer Lösung, welche auf $50 \mathrm{ccm} 2.8625 \mathrm{~g}$ Tellursäure gelöst enthielt.

3. Die Kapazität des Widerstandsgefälses wurde, wie üblich, mit $1 / 50^{-n o r m}$. Kaliumchloridlösung gemessen und $\mathrm{zu} k=118.17$ gefunden.

4. Gefunden wurden nach der Kohlradsch'schen Methode• folgende Werte:

$$
t=25^{0} .^{1}
$$

\begin{tabular}{|c|c|c|c|c|}
\hline I. & $\begin{array}{c}v \\
16\end{array}$ & $\begin{array}{c}a \\
642\end{array}$ & $\begin{array}{c}w \\
10000\end{array}$ & $\begin{array}{c}\mu \\
0.3390\end{array}$ \\
\hline \multirow{4}{*}{ II. } & 4 & 270.3 & 1000 & $0.17 \pm 82$ \\
\hline & 8 & 192.0 & 1000 & 0.22470 \\
\hline & 16 & 634.5 & 10000 & 0.32820 \\
\hline & 32 & 530.0 & 10000 & 0.42640 \\
\hline \multirow{9}{*}{ III. } & 4 & 270.0 & 1000 & 0.17480 \\
\hline & 8 & 184.5 & 1000 & 0.21400 \\
\hline & 16 & 615.0 & 10000 & 0.30200 \\
\hline & 32 & 500.0 & 10000 & 0.37820 \\
\hline & & also in & & \\
\hline & 4 & 270.15 & 1000 & 0.17480 \\
\hline & 8 & 188.25 & 1000 & 0.21930 \\
\hline & 16 & 624.75 & 10000 & 0.32050 \\
\hline & 32 & 515.00 & 10000 & 0.40230 \\
\hline
\end{tabular}

${ }^{1} t=$ Temperatur; $v=$ Verdünnung; $a=$ Stellung des Kontakträdchens; $w=$ Widerstand, ausgedrückt in Ohm; $\mu=$ molekulare Leitfähigkeit. 
Grölsere Verdünnungen als $v=32$ zu messen, war mir leider nicht möglich, da die hierzu nötigen Widerstandsgefälse nicht zur Verfügung standen.

Vergleicht man nun die von mir für $\mu$ gefundenen Zahlenwerte mit denen, die für die anderen Säuren der Schwefelgruppe angegeben sind, so bemerkt man einen ganz erheblichen Unterschied, denn für die Selensäure fand Ostwald, bei $25^{\circ}$ und $v=3 \longleftarrow$, den Wert 539, und KoHLrausch bestimmte die Leitfähigkeit einer $50 \%$ igen Schwefelsäure bei $78^{\circ}$ zu 370. Dagegen findet man bei Berücksichtigung der schwachen Säuren, dafs die Teliursäure ihrem Säurecharakter nach ein völliges Analogon des Schwefelwasserstoffs und der Cyanwasserstoffsäure ist, deren Leitfähigkeiten, von OsTWALD bei $25^{\circ}$ und einer 32 fachen Verdünnung bestimmt, die mit meinen Resultaten gut übereinstimmenden Zahlen: $\mathrm{H}_{2} \mathrm{~S}$ zu 0.91 und $\mathrm{HCN}$ zu 0.46 ergaben.

\section{Bestimmung der äquivalenten Leitfähigkeit des neutralen Kalium- tellurats.}

1. Benutzt wurde ein Präparat, welches aus einem Gemisch von reinster Tellursäure und reiner, frisch bereiteter Kalilauge durch Alkohol gefällt und, zur Entfernung etwa anhaftender Kalilauge, öfters mit reinem Wasser ausgewaschen worden war. Die Krystalle wurden bis zur Gewichtskonstanz in einem mit Chlorcalcium angefüllten Exsikkator getrocknet.

2. Angewandt wurden je $20 \mathrm{ccm}$ einer äquivalenten Kaliumtelluratlösung, d. b. einer solchen, die auf $100 \mathrm{ccm} 0.5625 \mathrm{~g} \mathrm{~K}_{2} \mathrm{TeO}_{4}+$ $5 \mathrm{H}_{2} \mathrm{O}$ enthielt.

3. Die Kapazität des Widerstandsgefälses wurde wieder mit $1 / 0^{-}$-norm. Kaliumchloridlösung bestimmt und $\mathrm{zu} 109.30$ gefunden.

4. Erhalten wurde folgende Zahlenwerte, bei denen unter $\lambda$ die äquivalente Leitfähigkeit zu verstehen ist:

(S. Tabelle, S. 10.)

Vergleicht man diese Werte mit den für andere neutrale Kalisalze zweibasischer Säuren gefundenen, so ergiebt sich, dafs sie sich einerseits von denen des neutralen Kaliumsulfats ziemlich weit entfernen, für die WALDEN bei $25^{\circ}$ und 1024 facher Verdünnung die Zahl 272.80 fand, während sie andererseits in ganz gute Übereinstimmung mit den für das neutrale Kaliumkarbonat experimentell gefundenen Werten zu bringen sind. 
$t=25^{\circ}$.

\begin{tabular}{|c|c|c|c|c|}
\hline & $v$ & $a$ & $w$ & 2 \\
\hline \multirow{8}{*}{ I. } & 32 & 781.50 & 100 & 125.10 \\
\hline & 64 & 663.00 & 100 & 137.60 \\
\hline & 128 & 511.0 & 100 & 146.20 \\
\hline & 256 & 347.019 & 100 & 148.70 \\
\hline & 512 & 213.00 & 100 & 151.50 \\
\hline & 1024 & 587.50 & 1000 & 159.40 \\
\hline & 32 & 780.50 & 100 & 124.60 \\
\hline & 64 & 663.00 & 100 & 137.60 \\
\hline \multirow[t]{11}{*}{ I1. } & 128 & 515.00 & 100 & 148.60 \\
\hline & 256 & 359.50 & 100 & 157.00 \\
\hline & 512 & 227.00 & 100 & 164.30 \\
\hline & 1024 & 600.50 & 1000 & 168.20 \\
\hline & & \multicolumn{2}{|c|}{ also ina Mittel: } & \\
\hline & 32 & 781.00 & 100 & 124.85 \\
\hline & 64 & 663.00 & 100 & 137.60 \\
\hline & 128 & 513.00 & 100 & 147.40 \\
\hline & 256 & 353.75 & 100 & 152.85 \\
\hline & 512 & 220.00 & 100 & 157.90 \\
\hline & 1024 & 594.00 & 1000 & 163.80 \\
\hline
\end{tabular}

In Übereinstimmung mit den soeben argegebenen Resultaten mufste nun auch der Fall eintreten, dafs die Tellursäure sich nicht unter Anwendung eines Indikators titrieren lassen würde, und ich fand diese Vermutung vollkommen bestätigt, als ich mir zur Darstellung des neutralen Kaliumtellurats, welches ich zu den Leitfähigkeitsbestimmungen benötigte, durch Titration mit Normallauge eine genaue neutrale Lösung darstellen wollte.

Die Tellursäure verhält sich Lackmustinktur gegenüber ganz genau so, wie die wässerige Lösung des Schwefeldioxyds, denn noch bevor die äquivalente Menge Normallauge zugegeben ist, geht die Farbe des Indikators langsam und stetig durch Violett in Blau über, ohne dals man den Farbenumschlag genau erkennen könnte. Dementsprechend zeigt auch die Lösung des neutralen Kaliumtellurats alkalische Reaktion.

Bei einigen Titrationsversuchen, die ich anstellte, am die Einwirkung der verschiedenen gebräuchlichen Indikatoren zu studieren, erhielt ich die günstigsten Resultate noch mit Phenolphtalern, indem der Farbenumschlag bei Anwendung von $20 \mathrm{ccm} 1 / 5$-norm. Tellur- 
säurelösung nach Zugabe von $16 \mathrm{ccm} 1 / 10$-norm. Natronlauge eintrat, während Lackmustinktur schon nach Zusatz ron $9 \mathrm{ccm}$ Lauge seine Farbe veränderte und Methylorange sowohl als auch Congo sich überhaupt als vollkommen ungeeignet erwiesen.

\section{Bestimmung der Gefrierpunktserniedrigung für Tellursäure.}

Obwohl schon durch die Leitfähigkeitsbestimmungen zur Evidenz bewiesen war, dafs die T'ellursäure nur zum T'eil in ihrer wässerigen Lösung in Ionen gespalten sei, sehien es mir doch noch ganz anlgebracht, auch die Gefrierpunltserniedrigung zu bestimmen, um genau feststellen zu können, dal's die 'Telhursäure als Nichtelelstrolyt auftritt.

Zur Ausführung dieser Bestimmungen mufste die Säure ebenfalls wieder in wässeriger Säure angewandt werden, da sie sich in den sonst gebräuchlichen Lösungsmitteln entweder gar nicht, oder nur teilweise und unter Zersetzung auflöst; es ergab sich nun hierbei, dals die Konstante $T$ des Wassers, in dem die Tellursäure gelöst war, 19 wie für jeden anderen Nichtelektrolyten, z. B. Harnstoff', betrug.

Für die Bestimmungen nach dem BeckmanN'schen Verfahren wurde dasselbe Präparat der Säure und dasselbe reinste Wasser wie zu den Leitfähigkeitsbestimmungen benutzt, und ich erhielt dabei folgende Zahlen:

\begin{tabular}{|c|c|c|c|c|}
\hline & $S$ & $L$ & I) & $M^{1}$ \\
\hline 1 & $0.3212 \mathrm{~g}$ & $20.1388 g$ & $0.140^{\circ}$ & 218.50 \\
\hline 2 & 0.8715 & 20.1388 & 0.385 & 213.75 \\
\hline 3 & 0.4575 & 20.0008 & 0.190 & 228.70 \\
\hline 4 & 0.3405 & 20.1000 & 0.160 & 201.40 \\
\hline
\end{tabular}

Wie ersichtlich, haben diese Bestimmungen unter Benutzung des Wertes $I=19$ für die Konstante des Wassers ein Molekulargewicht der Tellursäure von $215 \mathrm{im}$ Mittel ergeben; und dies ist ein Wert; der mit dem für $\mathrm{H}_{6} \mathrm{TeO}_{6}$ zu 229.6 berechneten vorzüglich übereinstimmt.

${ }^{1} S=$ angewandte Substanz in Grammen; $L=$ Gewicht des als Lösungsmittel benutzten Wassers in Grammen; $D=$ beobachtete Gefrierpunktserniedrigung; $M=$ gefundenes Molekulargewicht. 
Und somit hat denn auch diese Bestimmung emeut den Beweis dafür erbracht, dals die Tellursäure zu den schwächsten, uns bekannten Säuren zu zählen ist.

Aus dem merkwürdigen Verhalten der Tellursäure bei den oben beschriebenen Versuchen scheint mir hervorzugehen, dals sie nicht die Konstitutionsformel:

$$
\mathrm{HO}-\overbrace{\mathrm{Te}}-\mathrm{OH}+2 \mathrm{H}_{2} \mathrm{O}=\mathrm{H}_{2} \mathrm{TeO}_{4}+2 \mathrm{H}_{2} \mathrm{O}
$$

besitzt, sondern, dal's ihr weit eher die Formel:

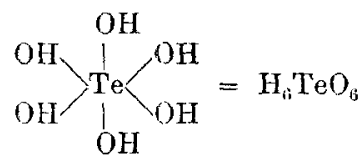

zukommt; denn meiner Ansicht nach läfst sich nur durch die zweite Formel die Eigenschaft der Säure, beim Austreiben des Wassers gleichzeitig mit Sauerstoff abzugeben, erklären.

Es ist sehr wahrscheinlich, daf́s die beiden Moleküle Wasser nicht als Krystall-, sondern als Konstitutionswasser zu betrachten sind, und es sprechen für diese Annahme folgende Gründe:

1. verliert die Säure gleichzıitig mit dem zweiten Moleküle des ihr zugehörigen Wassers Sauerstoff;

2. verhält sich die Substanz, welche von den älteren Forschern als $\mathrm{H}_{2} \mathrm{TeO}_{4}$, von mir aber als ein Gemenge von $\mathrm{H}_{6} \mathrm{TeO}_{6}$ mit $\mathrm{TeO}_{3}$ angesprochen wird, äulserst indifterent gegen chemische Agentien und scheidet sich, wenn sie in Reaktion gebracht ist, nur wieder als $\mathrm{H}_{6} \mathrm{TeO}_{6}$ bezw. in Gestalt deren Derivate $\mathrm{ab}$;

3. verwandelt sich das $\mathrm{Hydrat} \mathrm{H}_{2} \mathrm{TeO}_{4}+6 \mathrm{H}_{2} \mathrm{O}$ mit der grölsten Leichtigkeit in die Säure $\mathrm{H}_{6} \mathrm{TeO}_{6}$;

4. ist die Tellursäure nicht esterifizierbar, ${ }^{1}$ da man hierbei stets - nach den uns zur Verfügung stehenden Methoden - zu Derivaten der Säure $\mathrm{H}_{2} \mathrm{TeO}_{4}$ gelangen mülste, welch' letztere aber sicher ebensowenig existieren werden, als die Säure selbst;

1 Ich werde hierüber, sowie äber die Eigenschaften der Tellurate in einer späteren Abhandlung berichten. 
5. müfste bei den Bestimmungen der Gefrierpunktserniedrigung doch auch ein Wert für das Molekulargewicht der Säure gefunden worden sein, der kleiner als der $\mathrm{H}_{2} \mathrm{TeO}_{4}=193.6$ berechnete hätte sein müssen, da man ja bekanntlich bei diesen Methoden stets Zahlen zu erhalten pflegt, welche zwischen +20 und -20 des berechneten Wertes liegen.

Ich stehe daher nicht an, der Tellursäure die Konstitutionsformel

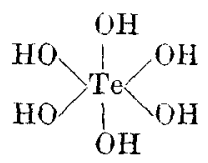

zuzuerteilen.

\section{Zusammenfassung der Resultate.}

1. Es ist zu empfehlen, das ungarische Rohtellur nicht als solches nach der von Staudenmaier angegebenen Methode auf 'Tellursäure zu verarbeiten, sondern das Tellur erst aus dem rohen Produkte abzuscheiden.

2. Die Methode, die Tellursäure in Gestalt ihres Baryumsalzes abzuscheiden, ist zu verwerfen.

3. Die von Berzelius beschriebene Säure $\mathrm{H}_{2} \mathrm{TeO}_{4}$ existiert nicht; sie ist ein Gemenge von $\mathrm{H}_{6} \mathrm{TeO}_{6}$ mit $\mathrm{TeO}_{3}$, öfters mit $\mathrm{TeO}_{2}$ verunreinigt.

4. Die Tellursäure giebt beim Erhitzen bis auf $145^{\circ}$ Wasser $a b$; der hierbei erreichte Gewichtsverlust entspricht jedoch nicht dem für $2 \mathrm{H}_{2} \mathrm{O}$ berechneten; über diese Temperatur erhitzt, giebt die Säure gleichzeitig mit dem letzten Anteile des Wassers schon Sauerstoff $\mathbf{a b}$ und verwandelt sich in das sub 3 beschriebene Gemenge

5. Die Tellursäure ist dimorph und krystallisiert sowohl nach dem regulären als auch nach dem hexagonal-rhomboëdrischen Systeme.

6. Aus den angeführten Leitfähigkeitsbestimmungen ist zu entnehmen, dafs die Tellursäure eine sehr schwache Säure, d. h. in Lösung nur zum geringsten Teile dissoziiert ist; sie steht auf gleicher Stufe mit Schwefelwasserstoff und Cyanwasserstoffsäure; die für die Leitfähigkeit des neutralen Kaliumtellurats gefundenen Zahlenwerte zeigen ebenfalls einen erheblichen Unterschied von den anderen Säuren der Schwefelgruppe. 
7. In ibrem Verhalten bei der Titration mittels Indikatoren ist die Tellursäure ein völliges Analogon der wässerigen Lösung des Schwefeldioxyds.

8. Durch die Bestimmung der Gefrierpunktserniedrigung für Tellursäure ist bewiesen worden, dals die Säure ein Nichtelektrolyt ist.

9. Der Tellursäure kommt nicht die Konstitutionsformel $\mathrm{H}_{2} \mathrm{TeO}_{4}+$ $2 \mathrm{H}_{2} \mathrm{O}$, sondern die Formel $\mathrm{H}_{6}{ }^{r} \mathrm{TeO}_{6} \mathrm{zu}$, d. h. die beiden Moleküle Wasser sind als Konstitutionswasser zu betrachten.

München, Anoryanisch-chem. Luboratorium der königl. teehn. Hochschule, August 1901.

Bei der Redaktion eingegangen am 23. September 1901. 